\title{
Molecular mechanisms of retinoid actions in skin
}

\author{
GARY J. FISHER ${ }^{1}$ AND JOHN J. VOORHEES
}

Department of Dermatology, University of Michigan Medical Center, Ann Arbor, Michigan 48109-0528, USA

\begin{abstract}
For more than 40 years, it has been appreciated that vitamin $A$ is a critical regulator of growth and differentiation of developing and adult mammalian and avian skin. Vitamin $A$ deficiency and hypervitaminosis $A$ cause disruption of normal cellular homeostatic mechanisms, resulting in impairment of skin barrier function. More recent studies demonstrating all-trans retinoic acid as the major biologically active form of vitamin $A$, and nuclear retinoid receptors as the major mediators of all-trans retinoic acid actions, have provided exciting new insights into the molecular basis of vitamin A actions. These recent insights have been the driving force for important advances in the many areas of retinoid research made during the past 6 years. Nowhere has this new knowledge been more extensively applied than toward understanding the molecular basis of retinoid physiology and pharmacology in skin. This article will review these recent findings and attempt to synthesize their meaning to provide a view into the mechanisms whereby retinoids participate in regulation of skin function.-Fisher, G. J., Voorhees, J. J. Molecular mechanisms of retinoid actions in skin. FASEB J. 10, 1002-1013 (1996)
\end{abstract}

Key Words: retinoic acid receptors $\cdot$ activator protein-1 $\cdot$ keratinocytes

\section{BACKGROUND}

\section{Morphology of human skin}

The two major compartments of human skin are epidermis and dermis (Fig. 1, left panel). The major cell type in the epidermis is the keratinocyte. Keratinocytes in the bottom (basal) layer undergo cell division, migrate upward through suprabasal layers, and undergo terminal differentiation to form the protective barrier. Melanocytes, which synthesize pigment and give skin its color, also reside in the bottom layer of the epidermis. The epidermis is separated from the dermis by the basement membrane zone composed primarily of type IV and type VII collagens, laminin, entactin, fibronectin, and proteoglycans. The dermis is primarily extracellular matrix, composed of type I and type III collagens, and elastin, which are synthesized by dermal fibroblasts. Blood supply to the skin occurs via capillaries in the dermis. A single topical application of $0.1 \%$ all-trans retinoic acid (tRA) ${ }^{2}$ for 4 days stimulates keratinocyte proliferation, increasing the numbers of epidermal cell layers and increasing epidermal thickness $(1,2)$ (Fig. l, right panel). tRA also causes characteristic compaction of the barrier and widening of spaces between keratinocytes. Although, for a variety of technical reasons, more is known about the effects of retinoids on epidermis than on dermis, cells in the dermis are also important targets for retinoid actions in skin.

\section{NUCLEAR RETINOID RECEPTORS AND THEIR TARGET GENES IN SKIN}

\section{Transgenic mouse models}

Retinoic acid receptors (RARs) and retinoid X receptors (RXRs) are members of the steroid hormone receptor superfamily of ligand-activated transcription factors (3). RAR- $\alpha, \beta$, and $\gamma$ RXR- $\alpha, \beta$, and $\gamma$ bind as heterodimers to specific DNA sequences (retinoic acid response elements, RARE) in regulatory regions of target genes (Fig. 2 , upper panel). Binding of tRA by RAR- $\gamma$ activates the heterodimeric complex (the $\mathrm{RXR} \alpha$ ligand 9-cis retinoic acid does not contribute to activation of liganded RAR- $\gamma$ in human skin), thereby stimulating target gene transcription by a process known as transactivation. Physiological actions of retinoids are believed to be mediated primarily through RAR/RXR-mediated transactivation.

Targeted disruption of RARs, through homologous recombination, has been used as a genetic approach to elucidate physiological functions of RARs in the mouse, as reviewed in ref 4 . The skin of mice null for either RAR- $\alpha$ (5) or RAR- $\gamma$ (6) appears phenotypically normal. Disruption of both RAR- $\alpha$ and RAR- $\gamma$, although resulting in severe developmental defects in many organs, surprisingly

\footnotetext{
${ }^{\mathrm{I}}$ To whom correspondence and reprint requests should be addressed, at: Department of Dermatology, Kresge I, R6558, University of Michigan Medical Center, Ann Arbor MI 48109-0528, USA.

2Abbreviations: AP-1, activator protein-1; ARAT, acyl CoA:retinol acyltransferase; CAT, chloramphenicol acetyltransferase; COUP-TF, chicken ovalbumin upstream promoter-transcription factor; CRBP, cellular retinol binding protein; CRABPII, cellular retinoic acid binding protein II; LRAT, lecithin:retinol acyltransferase; RAR, retinoic acid receptor; $R X R$, retinoid $X$ receptor; $R A R E$, retinoic acid response element; RXRE, retinoid X receptor element; tRA, all-trans retinoic acid.
} 
does not significantly alter epidermal morphology (7). However, additional functional and molecular characterization of the skin of these mice is clearly required and may reveal significant alterations not manifest at the morphological level. In contrast, targeted overexpression of dominant negative mutant RAR- $\alpha$ to mouse epidermis results in distinct phenotypic abnormalities. Overexpression of mutated RAR- $\alpha$ in suprabasal keratinocytes results in reduced barrier function due to defects in lipid deposition by keratinocytes undergoing terminal differentiation (8). No generalized defects in programmed differentiation of suprabasal keratinocytes are observed. Overexpression of dominant negative mutant RAR- $\alpha$ in basal keratinocytes results in dramatic impairment of epidermal maturation (9). Even though these results are indicative of RAR involvement in normal skin development and function, caution must be exercised in their interpretation, as the dominant negative RAR- $\alpha$ mutants that were used are capable of heterodimerizing with RXRs. Therefore, through sequestration of RXRs, the mutant RAR- $\alpha$ could interfere with functions of other RXR heterodimeric partners, in addition to RARs (see below). Thus, one cannot conclude that the observed phenotypic alterations in skin were due exclusively to interference with RAR function.

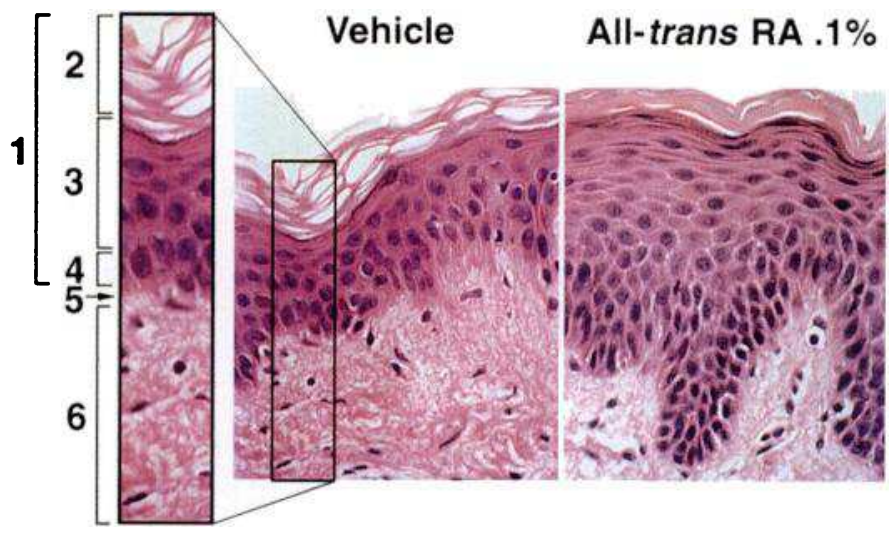

Figure 1. Histology of human skin treated with vehicle or $0.1 \%$ tRA. The two major compartments of human skin are epidermis (\#1) and dermis (\#6). The major cell type in the epidermis is the keratinocyte. Keratinocytes in the bottom (basal) layer (\#4) undergo cell division, migrate upward through suprabasal layers (\#3), and undergo terminal differentiation to form the protective barrier (\#2). Melanocytes, which synthesize pigment and give skin its color, also reside in the bottom layer of the epidermis (not apparent in these sections of skin). The epidermis is separated from the dermis by the basement membrane zone (\#5). The dermis (\#6) is primarily extracellular matrix, composed of collagens and elastin that are synthesized by sparse dermal fibroblasts. Blood supply to the skin occurs via capillaries (not apparent in these sections of skin) in the dermis. A single topical application of $0.1 \%$ tRA for 4 days stimulates keratinocyte proliferation, causing increased numbers of epidermal cell layers and increased epidermal thickness (right panel). tRA also causes characteristic compaction of the barrier, and widening of the spaces between keratinocytes. \#1: Epidermis (keratinocytes); \#2: nonliving barrier (cross-linked envelopes and lipids); \#3: suprabasal keratinocyte layers (differentiation); \#4: basal keratinocyte layers (proliferation); \#5: dermoepidermal junction (basement membrane zone); \#6: dermis (collagen and fibroblasts.

\section{Nuclear retinoid receptors}

Human epidermis expresses transcripts for RAR- $\alpha$, RAR- $\gamma$, RXR- $\alpha$, and RXR- $\beta$ (10). Transcripts for RAR- $\beta$ are low or undetectable, whereas RXR- $\gamma$ mRNA is undetectable. A similar pattern is seen in cultured human keratinocytes and dermal fibroblasts (11). Unlike other cell types, RAR- $\beta$ expression is not induced by tRA in human keratinocytes in vivo or in culture (11). This is not due to failure of the RAR- $\beta 2-R A R E$ to function in keratinocytes, because in transient transfections the isolated RAR- $\beta 2$ RARE confers induction by TRA on a chloramphenicol acetyltransferase (CAT) reporter (12). In contrast to keratinocytes, RAR- $\beta$ is induced by tRA in cultured human dermal fibroblasts $(11,13)$.

Using ligand binding and immunological assays, total and individual RAR and RXR protein levels have also been determined in human epidermis (14). Total RXR protein levels are fivefold greater than RAR total protein levels. Among RXR proteins, nearly $90 \%$ is RXR- $\alpha$, with RXR- $\beta$ minimally detectable and RXR- $\gamma$ undetectable. Thus, among RAR proteins, nearly $90 \%$ is RAR- $\gamma$, with approximately $10 \%$ RAR- $\alpha$ and no detectable RAR- $\beta$. Relative levels of individual RXR and RAR proteins are in good agreement with their apparent relative mRNA levels. If RARs and RXRs are uniformly expressed in epidermal cells, approximately 1800 RARs and 9400 RXRs will be present in each cell. Thus, RXRs do not seem to be limiting for heterodimerization with RARs. This assumes, however, that the number of different RXR partners is limited and that they are expressed at levels similar to, or less than, RARs (Fig. 2, lower panel). So far, this appears to be the case in human epidermis. Levels of RXR partners for which ligands are known, i.e., thyroid hormone receptor, vitamin $D$ receptor, and peroxisome proliferator-activated receptor, appear to be relatively low compared to RARs. Levels of other known RXR partners such as LXR (15), NGFI-B (16), NURR1 (16), RIP14 (17), and RIP15 (17), and other unknown orphan receptors that potentially might heterodimerize with RXRs, in human skin, however, are not known. The extent to which competition among nuclear receptors for interaction with RXRs influences RAR-mediated responses in skin remains to be elucidated.

\section{Retinoic acid receptor- $\gamma$ preferentially binds tRA}

RARs in human epidermis bind all-trans and 9-cis retinoic acid with similar high affinity (14). Equilibrium dissociation constants $\left(K_{d}\right)$ for binding of these two ligands by human epidermal RARs are similar to those reported for recombinant RARs (0.2-0.7 nM) (18). Allenby et al. (19) reported that although the off-rates for tRA binding to RAR- $\alpha$, RAR- $\beta$, and RAR- $\gamma$ are similar, off-rates for 9-cis retinoic acid differ among the three RARs, with RAR- $\beta$ the slowest and RAR- $\gamma$ the fastest. As a consequence of this, in the presence of mixtures of all-trans and 9-cis retinoic acid, RAR- $\gamma$ strongly prefers tRA. 
RAR- $\gamma$, in human skin nuclear extracts and intact epidermal cells, displays a similar strong preference for alltrans vs. 9-cis retinoic acid. At ratios of up to 4 to 1 (9-cis retinoic acid to tRA), no binding of 9-cis retinoic acid is detectable. This is in clear distinction to RAR- $\alpha$ in human skin, which when incubated with mixtures of all-trans and 9-cis retinoic acid in differing ratios, binds the two ligands in the same proportions as are present in the mixtures.

These results suggest that only under conditions where 9-cis retinoic acid levels exceed tRA levels several fold would 9-cis retinoic acid be able to activate RAR- $\gamma$-mediated responses in human skin. In normal human skin, 9cis retinoic acid levels are less than tRA levels (20), thus precluding 9-cis retinoic acid as a physiological activator of RAR- $\gamma$. 9-cis Retinoic acid applied topically to human skin is rapidly isomerized to tRA (20). The tRA formed would be expected to displace any 9-cis retinoic acid bound to RAR- $\gamma$. Because RAR- $\gamma$ comprises $90 \%$ of total RARs in human skin, 9-cis retinoic acid probably serves a minor, if any, role in regulating RAR-mediated responses in human skin either under physiological or pharmacological conditions.

\section{Retinoic acid receptor/retinoid $X$ receptor heterodimers}

In gel shift studies, RARs and RXRs in nuclear extracts from human skin and cultured keratinocytes bind exclusively as heterodimers to RARE, DNA sequences composed of hexameric direct repeats spaced by two (DR-2) or five nucleotides (DR-5), and to retinoid $X$ response

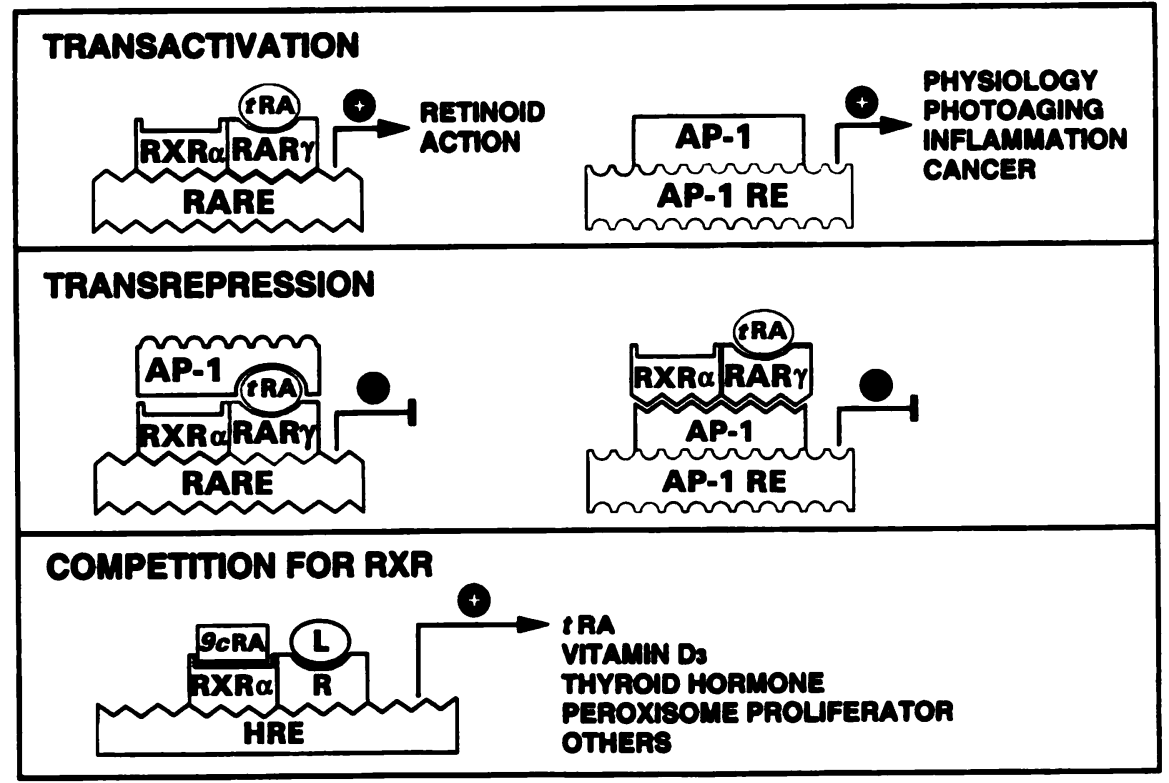

Figure 2. Functional interactions of retinoid receptors in human skin. Retinoic acid receptor- $\gamma$ (RAR- $\gamma$ ) and retinoid X receptor- $\alpha(\mathrm{RXR} \alpha)$ are the predominant retinoid receptors in human skin. Top panel: RAR- $\gamma$ and RXR $\alpha$ bind as heterodimers to specific DNA sequences (retinoic acid response elements) in regulatory regions of target genes. Binding of tRA by RAR- $\gamma$ activates the heterodimeric complex (the RXR $\alpha$ ligand 9-cis retinoic acid does not contribute to activation of liganded RAR- $\gamma$ in human skin), thereby stimulating target gene transcription by a process known as transactivation. Physiological actions of retinoids in skin are mediated primarily through RAR/RXR-mediated transactivation. Middle panel: Retinoid receptors also interact with transcription factor AP-1, composed of jun and fos proteins. AP-1 regulates transcription of many genes that participate in regulation of growth, differentiation, and responses to stress (physiology). Disregulation of AP-l is associated with malignant transformation, inflammatory diseases states, and sun-induced skin aging. Interaction of retinoid receptors with AP-1 is dependent on IRA, and the complex that is formed is unable to transactivate either RARE or AP-1 response element (AP-1 RE) -containing genes (transrepression). For simplicity, interaction between retinoid receptors and AP-1 is depicted as direct; although the exact nature of this complex (or complexes) is not known, it is most likely indirect and involves additional protein components. Transrepression of AP-1 appears to be a major mechanism of pharmacological anti-inflammatory and antiproliferative effects of retinoids. Lower panel: $R X R \alpha$ in skin also functions as a heterodimeric partner with other members of the steroid receptor superfamily $(R)$, including vitamin $\mathrm{D}$, thyroid hormone, and peroxisome proliferator-activated receptors. Thus, the interaction of RXR with these nuclear receptors limits the ability of RXR to bind to RARs. Each heterodimeric complex binds to specific hormone response elements (HRE) in target genes. Binding of cognate ligands (L) by these receptors stimulates transactivation, which is further enhanced for certain receptors, but not others, by the binding of 9-cis retinoic acid (9cRA) to $R X R \alpha$. 
elements (RXRE), direct repeats spaced by one nucleotide (DR-1) $(12,14,21)$. The presence of either all-trans or 9-cis retinoic acid has no effect on heterodimer DNA binding. Nuclear extracts, prepared from keratinocytes overexpressing RXRs, display RXR homodimer binding to DR-1, and this binding is enhanced by 9-cis retinoic acid (12). In keratinocytes, transcription of CAT reporter genes regulated by RAREs (DR-2 or DR-5) is induced by tRA, 9-cis retinoic acid, and the RAR-specific ligand CD367, but not the RXR-specific ligand SR11237. In contrast, transcription of an RXRE (DR-1) CAT reporter in keratinocytes is not activated by any of the above ligands. The inability of RAR/RXR heterodimers to transactivate DR-l elements in keratinocytes is consistent with results in other cell types, shown to be a consequence of the orientation the heterodimer adopts on a DR-1 element (22). The RXRE reporter, however, is induced by tRA (by isomerization to 9-cis retinoic acid), 9cis retinoic acid, and SR 11237 (but not CD367) in keratinocytes overexpressing RXRs, i.e., under conditions that promote RXR homodimers. Thus, at their physiological levels, RARs and RXRs in human skin and keratinocytes bind DR-2 and DR-5 RAREs as heterodimers and transactivate these elements in response to RAR, but not RXR, ligands (Fig. 2, upper panel). Lack of RXR homodimer formation and DR-1 transactivation in human keratinocytes is consistent with studies of mouse skin, where topical application of SR11237 evokes no detectable response (23).

\section{Retinoid receptor target genes}

Several genes have been shown to be directly regulated by RAREs through functional RAREs in their promoters (24). Of these, the best characterized in human skin are cellular retinoic acid binding protein II (CRABPII) (25, $26)$, cellular retinol binding protein (CRBP) (21), and keratin $6(27,28)$. Each of these genes is induced in human skin by tRA application. The human CRABPII gene contains a DR-5 RARE $5.6 \mathrm{~kb}$ upstream from the transcriptional start site that confers transcriptional induction by tRA (29). Thus, induction of CRABPII mRNA by synthetic retinoids can be used as an indicator of specific retinoid activity in human skin.

Although the human CRBP gene has not been characterized, CRBP in rat (30) and mouse (31) contains a functional DR-2 RARE. In human skin, the kinetics and retinoid dose dependence of CRBP mRNA induction parallel those for CRABPII, thus making it likely that transcription of human CRBP, like its rodent counterparts, is regulated by an RARE (21).

Keratin 6 normally is not expressed in human skin; keratin 6 is expressed, however, in hyperproliferative skin diseases and in response to retinoids. Only a limited region of the human keratin 6 promoter has been characterized (32). This region, however, is highly homologous to the bovine keratin 6 promoter, which has been more extensively studied (33). The bovine keratin 6 promoter contains a functional RARE located 540 bp upstream of the transcriptional start site. Although this region lies further upstream than has yet been studied in the human keratin 6 gene, it is likely that a homologous element is present in the human keratin 6 promoter and accounts for retinoid induction. The human and bovine keratin 6 promoters also contain positive regulatory elements for transcription factor AP-1 (activator protein-1). This element is probably responsible for keratin 6 induction in hyperproliferative skin conditions in which AP-1 is activated. The AP-1 element in the keratin 6 promoter is functionally antagonized by RARs (i.e., transrepressed). In spite of this, the net effect of retinoids is not inhibition of keratin 6 expression, but rather induction, due to the RARE in its promoter.

\section{AP-1 transrepression by retinoid receptors}

Functional antagonism of AP-1 by RARs, termed transrepression, is not unique to regulation of keratin 6 expression. Transrepression is a widespread mechanism for cross-talk between retinoid receptors and AP-1 $(34-36)$. The molecular mechanism of transrepression is believed to involve direct or indirect protein-protein interactions between retinoid receptors and AP-1 components $c$-jun and $c$-fos, and/or competition between retinoid receptors and $\mathrm{APl}$, for a common factor (or factors) required for their activities $(35,36)$ (Fig. 2, middle panel). Like transactivation, transrepression is dependent on tRA. An example with considerable practical importance of transrepression has been described in the context of molecular responses of skin to sunlight. Chronic exposure to ultraviolet irradiation from the sun causes premature skin aging, referred to as photoaging (37). Photoaging is manifested by wrinkles, reduced elasticity, fine lines, darkened and uneven pigmentation, and roughness. The major histological features of photoaged skin are evident in connective tissue (dermis) underlying the epidermis. These include accumulation of disorganized elastin-containing fibers and reduced content of type I and type III procollagens in the extracellular matrix. These findings have led to the conclusion that the cause of photoaging is sun-induced dermal damage $(37,38)$. This damage is likely mediated by destruction of the extracellular matrix of the dermis by matrix-degrading metalloproteinases (39).

Promoters for these metalloproteinases such as interstitial collagenase, gelatinase $\mathrm{B}$, and stromelysin I contain AP-1 response elements, which are necessary for gene transcription. Exposure of human skin to doses of ultraviolet irradiation equivalent to a few minutes of sunlight up-regulates AP-1 and induces metalloproteinase transcripts, proteins, and activities (39). It is believed that the ensuing dermal damage is not perfectly repaired, resulting in an invisible scar. Accumulation of such solar invisible scars, caused by repeated sunlight exposure over many years, eventuates in visible solar scars, which are responsible for photoaging (39). 
Treatment of human skin with tRA or its metabolic precursor all-trans retinol prior to ultraviolet irradiation exposure blocks AP-1 activation and induction of interstitial collagenase, gelatinase $B$, and stromelysin I (39). Inhibition of matrix metalloproteinase induction by tRA presumably occurs through transrepression of AP-1, because tRA prevents ultraviolet irradiation-induced AP-1 activation (39). These data suggest that tRA and all-trans retinol may be beneficial for prevention of photoaging, although this has not yet been tested in prospective clinical trials designed to detect prevention. In addition, tRA has been shown to improve the appearance of photoaged skin (40). This may well occur, at least in part, as a result of restitution by IRA of type I collagen synthesis, which is reduced in photoaged skin (41-43). Thus, tRA, acting through two apparently separate mechanisms, may be useful both for preventing photoaging and for repairing photoaging once it has occurred.

\section{RETINOID METABOLISM IN HUMAN SKIN}

\section{Retinol esterification}

Retinyl esters and $\beta$-carotene taken in from the diet are converted to all-trans retinol in the intestine and stored in the liver after reconversion to retinyl esters. Retinol released from the liver is transported in the circulation complexed to plasma retinol binding protein. Circulating retinol apparently is taken up by skin cells through passive diffusion. In human skin, all-trans retinol is metabolized to at least four important products: retinyl esters, 14-hydroxy-4,14-retro-retinol, all-trans 3,4-didehydroretinol (and its ester), and tRA. Among these three metabolites, retinyl ester levels are greatest. Retinyl esters function as a storage form of retinol. Their hydrolysis yields free retinol, which can be further metabolized.

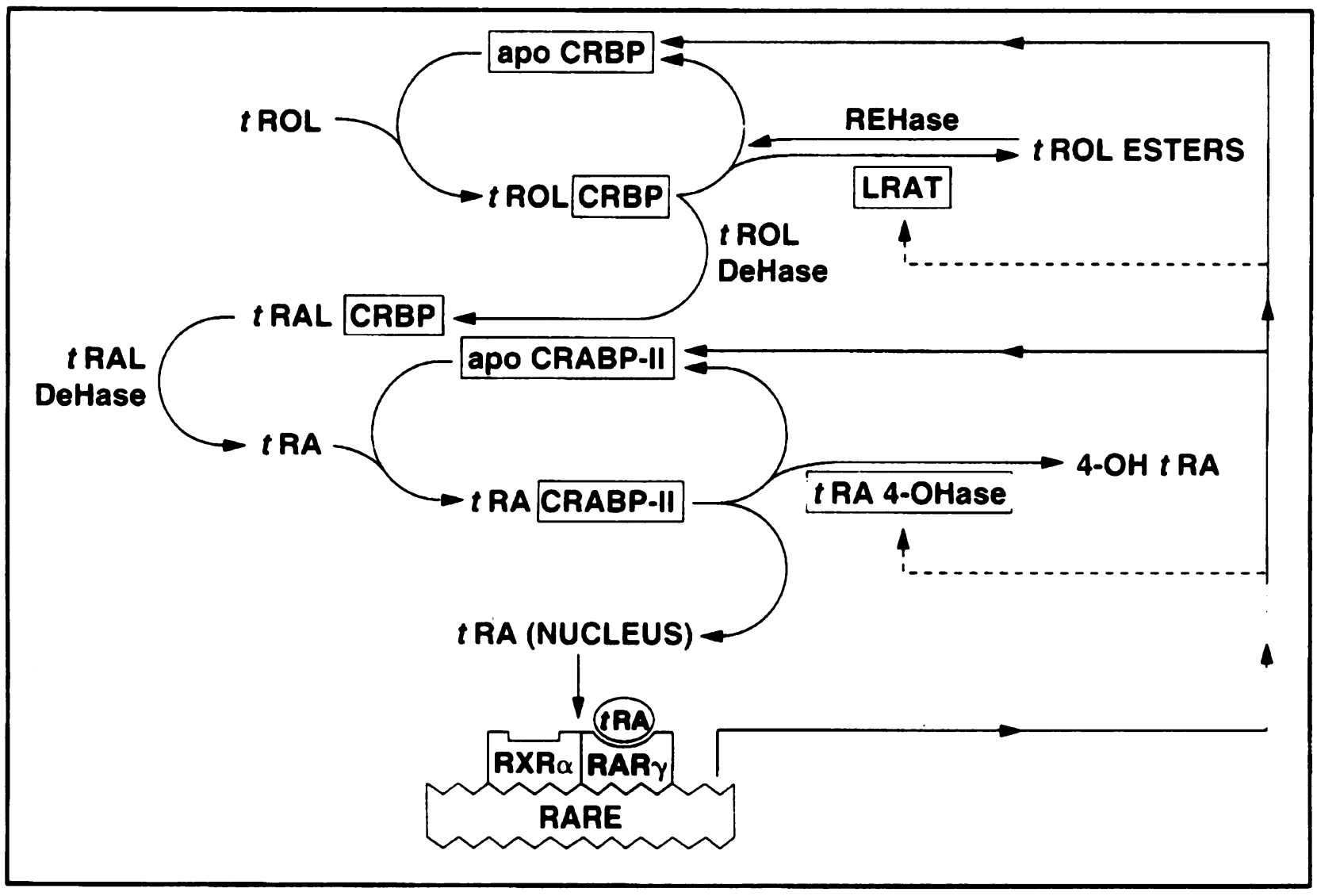

Figure 3. Autoregulation of tRA (tRA) metabolism in human skin. All-trans retinol (tROL), taken up from the circulation or applied externally, is bound by cellular retinol binding protein (CRBP) in keratinocytes. tROL bound to CRBP is either esterified to retinyl esters (tROL ESTERS), catalyzed by lecithin:retinol acyltransferase (LRAT) or sequentially oxidized to all-trans retinaldehyde (tRAL), catalyzed by retinol dehydrogenase (tROL DeHase), and then to all-trans retinoic acid (tRA), which is catalyzed by retinaldehyde dehydrogenase (tRAL DeHase). IRA, synthesized from tROL or applied topically, binds to cellular retinoic acid binding protein-II (CRABP-II). tRA bound to CRABP-II is either hydroxylated by tRA 4-hydroxylase (tRA 4-OHase) to 4-hydroxy tRA (4-OH tRA), which has only weak biological activity, or enters the nucleus where it binds to and activates the RAR- $\gamma$ component of retinoid $X$ receptor $(R X R \alpha) /$ retinoic acid receptor (RAR- $\gamma)$ heterodimers. Activation of RXR $\alpha /$ RAR- $\gamma$ by tRA stimulates transcription of genes containing retinoic acid response elements (RARE) in their promoters. tRA induces CRBP, CRABP-II, LRAT, and tRA 4-OHase (each shown in red) in human skin, and thereby tightly regulates its own level. CRBP and CRABP-II contain RAREs in their promoters, and therefore their transcription is directly stimulated by RXR/RAR. The mechanism (direct or indirect) through which tRA induces LRAT and tRA 4-OHase is not known (signified by dashed arrows), but may also involve RXR/RAR-mediated gene transcription. 
Topical treatment of human skin for $24 \mathrm{~h}$ with all-trans retinol or all-trans retinaldehyde increases retinyl ester levels greater than 10-fold in the living cell layers (44). Skin expresses both lecithin:retinol acyltransferase (LRAT) and acyl CoA:retinol acyltransferase (ARAT) activities, the two enzymes known to catalyze retinyl ester synthesis $(45,46)$. The normally low LRAT activity of cultured keratinocytes is induced by retinoids, probably via RARs, within $12 \mathrm{~h}$ (45) (Fig. 3). Induction of LRAT activity by retinoids increases retinyl ester formation and reduces tRA biosynthesis (45). Under these conditions, inhibition of LRAT by phenylmethylsulfonyl fluoride restores IRA synthesis. Thus, reduction of IRA synthesis in skin occurs through increased competition for all-trans retinol between LRAT and retinol dehydrogenase rather than by inhibition of tRA synthesis per se.

Regulation of LRAT activity by retinoids provides a mechanism of autoregulation of tRA synthesis through substrate availability. Under conditions of retinoid sufficiency, all-trans retinol esterification is induced and tRA synthesis is reduced, whereas under conditions of retinoid deficiency the opposite situation occurs (Fig. 3).

\section{Utilization of retinyl esters}

In human skin in vivo, retinol esterifying activity is fourfold greater, on a per cell basis, in keratinocytes in the bottom layer of the epidermis than in keratinocytes in upper layers (45). This suggests that retinoid levels are higher in the lower epidermis, which is close to the nutrient supply from capillaries in the high dermis, than in the upper epidermis. It also suggests that all-trans retinol stores, in the form of retinyl esters, are greater in keratinocytes in lower epidermal layers than in upper layers. Keratinocytes in the bottom layer of the epidermis migrate through the upper cell layers toward the outside. As they do so, they undergo maturation and terminal differentiation near the skin surface. Retinyl ester synthesis by keratinocytes in the bottom layer of the epidermis could provide these cells with a source of all-trans retinol during their migration and maturation. This scheme is consistent with the observation that maturing keratinocytes possess the capacity to metabolize all-trans retinol to its active metabolite tRA (47).

\section{tRA biosynthesis}

Biosynthesis of tRA from all-trans retinol occurs in two steps: all-trans retinol is oxidized to all-trans retinaldehyde, which is further oxidized to tRA (Fig. 3). Oxidation of all-trans retinol to all-trans retinaldehyde is rate limiting for tRA biosynthesis. Short-term topical treatment of human skin with all-trans retinol results in histological and molecular alterations that closely mimic those that occur in response to tRA (44). These include increased epidermal thickness due to increased keratinocytes proliferation, widening of intercellular spaces, compaction of the epidermal barrier, and induction of CRBP, CRABPII, and retinoic acid 4-hydroxylase (see below). tRA is minimally detectable in untreated and all-trans retinol-treated human skin $(44,47 \mathrm{a})$. Failure to detect significant accumulation of tRA in all-trans retinol-treated skin stems from two factors: 1) conversion of all-trans retinol to alltrans retinaldehyde is tightly regulated, and 2) tRA that is formed is rapidly hydroxylated by retinoic acid 4-hydroxylase. In cultured human keratinocytes, which do not express retinoic acid 4-hydroxylase activity, less than $1 \%$ of added all-trans retinol is converted to tRA (48). This low rate of conversion occurs despite large cellular accumulation of all-trans retinol, indicating that enzymatic capacity, rather than substrate availability, limits tRA synthesis. This limiting regulation occurs at the conversion of all-trans retinol to all-trans retinaldehyde, because in cultured keratinocytes, significantly greater tRA is formed from all-trans retinaldehyde than from all-trans retinol.

The observation that IRA and all-trans retinol elicit similar responses but that all-trans retinol does so without detectable increases in IRA levels indicates that endogenously synthesized tRA is much more efficient than exogenous IRA in activating retinoid pathways in human skin. This difference likely reflects limitations in cellular uptake and subsequent intracellular localization of exogenously applied tRA. These data suggest that all-trans retinol may be a more efficient and natural way to deliver tRA to the correct subcellular location within skin cells than direct treatment with tRA. The finding that all-trans retinol causes significantly less irritation, which is associated with retinoid excess, than IRA is consistent with this concept (49).

\section{4-Hydroxy-4,14-retro-retinol and all-trans 3,4-didehydroretinol biosynthesis}

In addition to converting all-trans retinol to retinyl esters and IRA, human epidermis also synthesizes 14-hydroxy4,14-retro-retinol (50). This metabolite of all-trans retinol was first shown to be biologically active in B-lymphocytes, where it substitutes for all-trans retinol in maintaining cell growth in culture (51). Addition of 14-hydroxy-4,14-retro-retinol to cultured keratinocytes does not alter their growth or differentiation. Similarly, topical treatment of human skin in vivo with 14-hydroxy4,14-retro-retinol causes no discernible cellular or molecular alterations (E. A. Duell, S. Kang, G. J. Fisher, and J. J. Voorhees, unpublished results). Therefore, although human skin possesses the capacity to synthesize 14-hydroxy-4,14-retro-retinol, the biological role of this retinoid in human skin remains obscure. Human skin also synthesizes all-trans 3,4-didehydroretinol (vitamin $A_{2}$ ) and its esters from all-trans retinol. The functional significance of this metabolic pathway is unknown. 


\section{tRA hydroxylation}

As mentioned, tRA in human skin is catabolized primarily to 4-hydroxy tRA (52). 4-Hydroxy tRA binds RARs with at least 100 -fold less affinity $(18,53)$ and is 10 -fold less potent in inducing retinoid responses in human keratinocytes and mouse skin than tRA (54). The gene encoding tRA 4-hydroxylase in human skin is unknown; however, its enzymatic properties, measured in human skin microsomes, are characteristic of members of the cytochrome P-450 superfamily (52). These include dependence on NADPH and inhibition by carbon monoxide and azole compounds. Several known P-450 isoforms that hydroxylate IRA at the $\mathrm{C} 4$ position can be distinguished from human skin tRA 4-hydroxylase by substrate specificity. Human skin tRA 4-hydroxylase is highly specific for tRA. It does not act on other retinoids, including alltrans retinol, all-trans retinaldehyde, and 9-cis and 13-cis retinoic acid (20), or on other nonretinoid substrates utilized by members of the $1 \mathrm{~A}, 2 \mathrm{~B}, 2 \mathrm{C}, 2 \mathrm{D}, 2 \mathrm{E}, 3 \mathrm{~A}, 4 \mathrm{~A}, 4$, and 5 cytochrome $P-450$ subfamilies. This strict substrate specificity is similar to that displayed by other members of the cytochrome P-450 family that participate in steroid hormone metabolism.

In untreated human skin, tRA 4-hydroxylase activity is minimally detectable. Activity is induced fivefold, however, after topical treatment with tRA (52) (Fig. 3). tRA 4-Hydroxylase activity is also induced in skin treated with 9-cis retinoic acid, 13-cis retinoic acid, and all-trans retinol.

Induction of tRA 4-hydroxylase by all-trans retinol is also likely mediated by tRA, which is formed by oxida- tion of all-trans retinol. Note that cellular and molecular responses of human skin to all-trans retinol are synergistically enhanced by liarozole, which inhibits tRA 4-hydroxylase (55). Thus, tRA 4-hydroxylase seems to function physiologically by regulating levels of endogenously synthesized tRA in human skin, in addition to its role in catabolizing pharamacological levels of exogenous tRA. Synergy between liarozole and all-trans retinol also supports the notion that actions of all-trans retinol in skin are mediated through its conversion to tRA.

\section{Autoregulation of $\mathrm{tRA}$ levels}

Note that expression of the four currently characterized proteins that control tRA homeostasis in human skin are regulated by tRA (Fig. 3). These include CRBP, CRABPII, LRAT, and tRA 4-hydroxylase. CRBP facilitates alltrans retinol uptake and presents it to LRAT for storage as retinyl ester (56). Similarly, CRABPII facilitates tRA uptake and presents it to tRA 4-hydroxylase for inactivation $(57,58)$. CRBP and CRABPII also participate in positive aspects of retinoid signaling. All-trans retinol bound to CRBP (holo-CRBP) is a substrate for conversion to tRA $(59,60)$, and apo-CRBP inhibits LRAT (56). Thus, the ratio of apo- to holo-CRBP is an important determinant regulating the balance between the oxidation and estrification of all-trans retinol (57). In addition, CRABPII may facilitate transport of tRA to the nucleus (61). Transcription of both the CRBP and CRABPII genes is positively regulated by RARs through RAREs in their promoters. Although neither tRA 4-hydroxylase nor LRAT has been cloned, it is tempting to speculate that

TABLE 1. Comparison of responses of keratinocytes to retinoids in culture vs. in vivo

\begin{tabular}{|c|c|c|}
\hline Response of Keratinocytes to Retinoids & In culture & In vivo \\
\hline \multicolumn{3}{|l|}{ Proliferation-related responses } \\
\hline Growth & Variable (64) & Increased $(1,2,27,28,72)$ \\
\hline Keratins 5 & Reduced (86) & No effect $(27,28)$ \\
\hline Keratin 14 & Reduced (86) & No effect $(27,28)$ \\
\hline Keratin 6 & Reduced (86) & Increased $(27,28)$ \\
\hline \multicolumn{3}{|l|}{ Differentiation-related reponses } \\
\hline Keratin 1 & Reduced (65) & No effect $(27,28)$ \\
\hline Keratin 10 & Reduced (65) & No effect $(27,28)$ \\
\hline Type 1 transglutaminase & Reduced $(67,68)$ & Increased (68) \\
\hline Loricrin & Reduced $(69,70)$ & Increased $(27,28)$ \\
\hline Involucrin & No change (87) & Increased (27) \\
\hline Profilaggrin/Filaggrin & Reduced (66) & Increased $(27,28)$ \\
\hline \multicolumn{3}{|l|}{ Other } \\
\hline CRABPII & No effect (26) & Increased (26) \\
\hline CRBP & No effect (21) & Increased (21) \\
\hline RA 4-hydroxylase & No effect ${ }^{b}$ & Increased (52) \\
\hline Collagenase induction & No effect ${ }^{c}$ & Reduced (39) \\
\hline
\end{tabular}


the genes for these two enzymes might also be directly regulated by RARs. This would provide a common mechanism for autoregulation of tRA levels (Fig. 3). Not yet accounted for is regulation of all-trans retinol dehydrogenase, the rate-limiting enzyme in tRA biosynthesis. Although cloning of this enzyme has been elusive, recent cloning of a specific all-trans retinol dehydrogenase from rat liver should substantially aid study of tRA biosynthesis in skin $(62,63)$. At present, it is evident that tRA, through coordinate regulation of retinoid binding proteins and metabolic enzymes, orchestrates a dynamic balance between its own synthesis and breakdown in human skin (Fig. 3).

\section{RESPONSES OF KERATINOCYTES TO RETINOIDS IN VIVO AND IN CULTURE DIFFER}

\section{Retinoids suppress terminal differentiation in cultured keratinocytes}

There are major differences in responses to retinoids between keratinocytes in culture and in vivo. This has led to some misconceptions concerning the physiological and pharmacological effects of retinoids on keratinocytes in vivo. Knowledge about differences between how keratinocytes respond to retinoids in culture vs. in vivo is made possible by the accessibility of skin in vivo to exogenous treatments and the relative ease with which it can be obtained. Obviously, most other tissues, especially in humans, cannot be directly obtained for study. Table 1 compares several responses of keratinocytes to retinoids in vivo and in culture. Paradoxically, retinoids often have effects on cultured keratinocytes opposite from those on keratinocytes in vivo. Differentiation of cultured keratinocytes can be divided into two sequential processes: 1) commitment, marked by irreversible growth arrest, and 2) functional differentiation, marked by expression of differentiation-specific gene products. Retinoids act predominantly on the latter process to inhibit expression of the differentiated phenotype (64). This is manifested as inhibition of expression of differentiation-associated keratins 1 and 10 (65), filaggrin (66), and several gene products associated with formation of the epidermal barrier (i.e., cross-linked envelopes) (67-71). Cross-linked envelopes consist of a network of specific proteins (loricrin, involucrin, cornifin) localized beneath the plasma membrane that are stabilized by cross-linkages with $\gamma$-glutamyl cysteinyl isopeptides. These protein cross-links are catalyzed by type I and type III transglutaminases. The highly insoluble cross-linked envelope serves as a critical structural element in the protective barrier of skin.

\section{Retinoids alter keratinocyte growth and differentiation in vivo}

In contrast, in vivo retinoids stimulate keratinocyte proliferation. This occurs with both topical and oral retinoid administration, indicating it is not simply a reaction to ir- ritation caused by topical treatment. In skin, keratinocytes in the bottom (basal) layer of the epidermis are capable of cell division. Basal keratinocytes migrate to upper (suprabasal) cell layers, and in so doing become committed to differentiation and lose the ability to divide. Whereas the effects of retinoids on keratinocyte growth in culture are variable and typicaly modest, retinoids consistently and strongly stimulate keratinocyte growth in vivo. Retinoids stimulate division of basal keratinocytes in vivo, which migrate upward and differentiate $(1,2$, 72). Growth stimulation by retinoids is dose dependent and results in increased numbers of suprabasal layers, populated by keratinocytes that exhibit precocious expression of differentiation-specific gene products $(27,28$, 68). These suprabasal keratinocytes form cross-linked envelopes and are sloughed off at the skin surface. Expression of keratin pairs 5 and 14 (made in basal keratinocytes) and of keratins 1 and 10 (made in suprabasal keratinocytes) is not altered by retinoid treatment in vivo $(27,28)$. Thus, retinoids do not suppress keratinocyte terminal differentiation in vivo, as is observed in culture. Rather, in vivo retinoids stimulate keratinocyte growth and modulate differentiation, and thereby modify the finely tuned balance between these two cellular processes. Retinoids do cause a modest decrease in barrier function. However, this effect does not reflect simply inhibition of cross-linked envelope formation, but rather more complex alterations involving increased keratinocyte turnover and altered differentiation.

\section{Retinoid receptor-dependent gene regulation in vivo vs. in culture}

In addition to disparate effects on keratinocyte growth and differentiation in vivo vs. in culture, several genes involved in retinoid metabolism that are retinoid inducible in vivo are not responsive to retinoids in culture. These include cellular retinoic acid binding protein II, cellular retinol binding protein, and retinoic acid 4-hydroxylase. Note that the former two genes contain RAREs in their promoters $(30,31)$, and are therefore regulated directly by nuclear retinoic acid receptors.

\section{Transactivation v8. transrepression}

Why do keratinocytes in vivo and in culture exhibit such strikingly dissimilar responses to retinoids? Accumulating evidence for cross-talk among retinoid and other signaling pathways, most notably transcription factor AP-1, may offer at least a partial explanation. Cross-talk among retinoid and other signaling pathways raises the possibility that responses of keratinocytes to retinoids may differ depending on the prevailing context of other signals. For example, under conditions of AP-1 activation, the predominant action of retinoids may be transrepression (i.e., inhibition of AP-1-driven responses), whereas under basal AP-1 conditions, transactivation of retinoid responsive genes may predominate. Likely candidates for signals 
present in vivo that are not present in culture are those derived from the dermis, which is known to have trophic influences on keratinocyte growth and maturation (73). These include soluble mediators such as keratinocyte growth factor (74) and insulin-like growth factor (75), as well as physical interactions of basal keratinocytes with structural proteins of the basement membrane.

\section{Nuclear retinoid receptor levels}

In addition to differences in dermal influences, keratinocytes in vivo and in culture differ in their levels of nuclear retinoid receptors. Cultured keratinocytes express approximately fourfold fewer retinoic acid receptors and sevenfold fewer retinoid $X$ receptors than keratinocytes in vivo (G. J. Fisher and J. J. Voorhees, unpublished results). Reduced receptor expression may limit the ability of cultured keratinocytes to respond to retinoids compared with keratinocytes in vivo. Clearly, unraveling the mechanisms that account for differences in retinoid responses between keratinocytes in vivo and in culture is a formidable proposition. It is equally clear that significant differences do exist, and that responses of cultured keratinocytes to retinoids cannot readily be extrapolated to keratinocytes in vivo.

\section{PERSPECTIVES}

Although probably more is known about retinoid physiology and pharmacology in skin than in any other single organ, much remains to be learned. The weight of evidence indicates that retinoid actions are primary mediated by retinoid receptors. If so, then how do retinoid receptors modulate cutaneous growth and differentiation? Given that retinoid receptors are transcription factors, this must be accomplished through regulation of gene expression. Knowledge of how retinoid receptors regulate gene expression and what genes are regulated is therefore critically important to unraveling how retinoids exert their effects in skin.

Based on current information, three distinct mechanisms exist through which retinoid receptors modulate gene expression: 1) transactivation through direct binding to RARE in target gene promoters, thereby stimulating basal transcriptional machinery (Fig 2, upper panel), 2) transrepression of AP-1 (and other transcription factors?) through protein-protein interactions (Fig. 2, middle panel), and 3) competition with other nuclear receptors (i.e., vitamin $\mathrm{D}$, thyroid hormone, and peroxisome proliferator activated) for heterodimerization with RXR (Fig. 2, lower panel).

\section{Transactivation and target genes}

Of these three mechanisms, transactivation of RARE-containing genes is the best established. At present, only three RARE-containing genes-CRABPII, CRBP, and keratin 6-have been demonstrated to be regulated by
tRA in skin. Because the products of these genes are not effectors, they cannot account for the pleiotypic effects of retinoids in skin. It is likely, therefore, that additional RARE-regulated genes will be identified that encode for proteins that more directly function to modulate cutaneous growth and differentiation. Possible candidates for such gene products include transcription factors, cell cycle components, kinases and phosphatases involved in cellular signaling, or master regulators of the above.

\section{Transrepression}

The second mechanism through which RARs modulate gene expression is transrepression of AP-1. Transrepression of AP-1 by tRA has been established as a pharmacological phenomenon. Whether it also operates as a physiological regulatory mechanism is unknown. However, one could imagine that under conditions of AP-1 activation, i.e., growth, differentiation, or stress, transrepression of AP-1 by RARs could act as a negative regulatory mechanism to protect against the consequences of inappropriately prolonged AP-1 activation. For this to occur would require coordinate regulation of tRA levels, through synthesis and/or uptake, and AP-1 activation, as transrepression is tRA-dependent. Whether this happens remains to be explored.

\section{Retinoid $\mathbf{X}$ receptor heterodimerization}

The third mechanism of RAR-mediated gene regulation is through competition of RARs with other nuclear receptors for heterodimerization with RXR. Sequestration of RXRs through heterodimerization with RARs could act to regulate negatively the activity of other nuclear receptors that partner with RXR in favor of RAR-mediated responses. Conversely, other RXR partners could negatively influence RAR activity. Competition among RARs and other nuclear receptors for $\mathrm{RXR}$ and/or its associated cofactors would be dictated by relative receptor levels and their affinities for RXR. Although such competition is theoretically possible, it has yet to be demonstrated in vivo.

\section{Effects of receptor selective synthetic retinoids}

Retinoids have been shown to be efficacious for several skin conditions, including psoriasis, acne, icthyosis, photoaging, and cancer, and to reduce skin atrophy caused by corticosteroid treatment for inflammatory diseases (76). Further development of synthetic retinoids for these and other conditions holds great promise. Skin is a therapeutic target particularly well suited for retinoids because of the possibility of topical application. Typically, systemic absorption of retinoids from topical administration is slow, thus minimizing the likelihood of unwanted systemic side effects. Retinoids that are selective for RAR subtype and for either RAR or RXR have been described $(34,77,78)$. Thus, in principle it is possible to design retinoids that exhibit a broad range of biological activi- 
ties. Given this possibility, how can these retinoids be best put to practical advantage? Part of the answer to this obviously complex question lies in whether RAR subtypes possess functional specificity. Although studies with transfected receptors acting over isolated response elements support the view that they do, this remains to be demonstrated in vivo.

RAR- $\gamma$ is the predominant RAR in skin. It is expressed in keratinocytes throughout the epidermis and in fibroblasts in the dermis (79). One would expect, therefore, that RAR- $\boldsymbol{\gamma}$-specific retinoids would have potent effects on skin cells. One drawback to their use, however, is the possibility of enhanced skin irritation and scaling, inasmuch as the intensity of these side effects of retinoids correlates with their ability to activate RAR- $\gamma(80)$. Whether RAR- $\alpha$, which is expressed at approximately one-tenth the level of RAR- $\gamma$, also mediates side effects is not known. Treatment of mouse skin with an RXR selective retinoid was without detectable effect (23), suggesting that RXR homodimers do not transactivate genes in skin. In contrast, RXR selective ligands can potentiate the actions of RXR-containing heterodimers in the presence of the ligand for the partner receptor.

\section{Anti-AP-1 synthetic retinoids}

Perhaps the most promising novel therapeutic uses of synthetic retinoids for skin diseases are as AP-1 antagonists. Immune cell activation and inflammation are typical features of many common skin diseases. Transcription of several lymphokine and cytokine genes, which drive the hyperplastic inflammatory response in skin, is regulated by transcription factors AP-l and NF- $\mathrm{KB}$. The antiinflammatory effects of corticosteroids, which are a mainstay of treatment for inflammatory skin diseases, are likely attributable to their ability to inhibit these transcription factors. Recent evidence indicates that retinoids, like corticosteroids, are able to inhibit AP-1 (35, 36). Unlike corticosteroids, however, retinoids do not inhibit NF-KB $(81,82)$. Retinoid inhibition of AP-1 is mediated by the interaction of RAR and RXR with jun and fos, which compose AP-1, to form an inactive complex. Thus, RARs possess two ligand-dependent functions: transactivation of genes containing RAREs, and transrepression of genes containing AP-1 response elements. tRA activates both functions. Some synthetic retinoids, however, are able to distinguish between these two functions. Synthetic retinoids have been described that exhibit potent AP-1 transrepression, but negligible RARE transactivation, and vice versa (83). Such retinoids should be useful in elucidating the roles of transactivation vs. transrepression in mediating physiological and pharmacological responses of skin to retinoids. In addition, retinoids that preferentially transrepress AP-1 may prove to be useful anti-inflammatory and antiproliferative therapeutic agents. Such retinoids also offer the intriguing possibility of reduced side effects, compared to conventional retinoids, due to their inability to stimulate
RAR (RAR- $\gamma$ is a mediator of retinoid irritation) transactivation activity.

\section{Negative regulation of retinoid signaling}

Finally, orphan receptors such as chicken ovalbumin upstream promoter-transcription factor (COUP-TF) and TAKl may function as negative regulators of retinoid signaling $(84,85)$. COUP-TF, which is expressed in skin, inhibits expression of RAR-dependent reporter genes in human keratinocytes. This most likely occurs as a result of competition of COUP-TF with RARs for RARE binding. COUP-TF binds not only to RAREs, but also to other direct repeat response elements recognized by vitamin $D$, thyroid hormone, and peroxisome proliferator-activated receptors. These receptors each function as heterodimers with RXR. Therefore, COUP-TF, which binds to DNA as homodimers, may influence the activities of the subset of nuclear receptors that partner with RXR. Obviously, there is much to learn about the function and regulation of COUP-TF and other orphan receptors in the skin. Information gained from these studies of the function and regulation of COUP-TF and other orphan receptors in the skin will likely provide important new insights into the physiology and pharmacology of retinoids in skin.

The authors would like to acknowledge Elizabeth Duell, Jia-Hao Xiao, J. T. Elder, Harvinder Talwar, Subhash Datta, Xiao-Yan Li, Charles Ellis, and Anders Astrom for their indispensible contributions to many of the studies cited in this review. Research described herein was supported in part by the Babcock Dermatological Research Fund, a research agreement with Johnson \& Johnson Corporation, and National Institutes of Health grant 5T32 AM07197-19.;

\section{REFERENCES}

1. Fisher, G. J., Esmann, J., Griffiths, C. E. M., Talwar, H. S., Duell, E. A., Hammerberg, C., Elder, J. T., Karabin, G. D., Nickoloff, B. J., Cooper, K. D., and Voorhees, J. J. (1991) Cellular, immunologic and biochemical characterization of topical retinoic acid-treated human skin. J. Invest. Dermatol. 96, 699-707

2. Weiss, J. S., Ellis, C. N., Headington, J. T., Tincoff, T., Hamilton, T. A., and Voorhees, J. J. (1988) Topical tretinoin improves photoaged skin. A doubleblind vehicle-controlled study. J. Am. Med. Assoc. 259, 527-532

3. Mangelsdorf, D. J., Thummel, C., Beato, M., Herrlich, P., Schutz, G., Umesono, K., Blumberg, B., Kastner, P., Mark, M., Chambon, P., and Evans, R. M. (1995) The nuclear receptor superfamily: The second decade. Cell 83, 835-840

4. Kastner, P., Mark, M., and Chambon, P. (1995) Nonsteroid nuclear receptors: What are genetic studies telling us about their role in real life? Cell 83, 859-870

5. Lufkin, T., Lohnes, D., Mark, M., Dierich, A., Gorry, P., Gaub, M.-P., LeMeur, M., and Chambon, P. (1993) High postnatal lethality and testis degeneration in retinoic acid receptor $\alpha(\mathrm{RAR} \alpha)$ mutant mice. Proc. Natl, Acad. Sci. USA 90, 7225-7229

6. Lohnes, D., Kastner, P., Dierich, P., Mark, M., LeMeur, M., and Chambon, $P$. (1993) Function of retinoic acid receptor- $\gamma(\mathrm{RAR} \gamma)$ in the mouse. Cell 73, $643-658$

7. Lohnes, D., Mark, M., Mendelsohn, C., Dolle, P., Dierich, A., Gorry, P., Gansmuller, A., and Chambon, P. (1994) Function of the retinoic acid receptors (RARs) during development. I. Craniofacial and skeletal abnormalities in RAR double mutants. Development 120, 2723-2748

8. Imakado, S., Bickenbach, J. R., Bundman, D. S., Rothnagel, J. A., Attar, J. S., Heyman, R. A., Evans, R. M., and Roop, D. R. (1995) Targeting expression of a dominant-negative retinoic acid receptor mutant in the expression of transgenic mice results in loss of barrier function. Genes \& $\mathrm{Dev}$. 9, 317-329 
9. Saitou, M., Sugai, S., Tanaka, T., Shimouchi, K., Fuchs, E., Narumiya, S., and Kakizuka, A. (1995) Inhibition of skin development by targeted expression of a dominant-negative retinoic acid receptor. Nature (London) 374, 159-162

10. Elder, J. T., Astrom, A., Pettersson, U., Tavakkol, A., Griffiths, C. E., Krust, A., Kaster, P., Chambon, P., and Voorhees, J. J. (1992) Differential regulation of retinoic acid receptors and binding proteins in human skin. J. Invest. Dermatol. 98, 673-679

11. Elder, J. T., Fisher, G. J., Zhang, 0. Y., Eisen, D., Krust, A., Kastner, P., Chambon, P., and Voorhees, J. J. (1991) Retinoic acid receptor gene expression in human skin. J. Invest. Dermatol. 96, 425-433

12. Xiao, J. H., Durand, B., Chambon, P., and Voorhees, J. J. (1995) Endogenous retinoic acid receptor-retinoid $X$ receptor heterodimers are the major functional forms regulating retinoid-responsive elements in adult human keratinocytes. J. Biol. Chem. 270, 3001-3011

13. Redfern, C. P., and Todd, C. (1992) Retinoic acid receptor expression in human skin keratinocytes and dermal fibroblasts in vitro. J. Cell Sci. 102, 113-121

14. Fisher, G. J., Talwar, H. S., Xiao, J. H., Datta, S. C., Reddy, A. P., Gaub, M. P., Rochette-Egly, C., Chambon, P., and Voorhees, J. J. (1994) Immunological identification and functional quantitation of retinoic acid and retinoid $X$ receptor protein in human skin. J. Biol. Chem. 269, 20629-20635

15. Mangelsdorf, D. J. (1995) LXR, a nuclear receptor that defines a distinct retinoid response pathway. Genes \& Dev. 9, 1033-1045

16. Perlmann, T., and Jansson, L. (1995) A novel pathway for vitamin A signaling mediated by RXR heterodimerization with NGFI-B and NURRI. Genes \& Dev. 9, 769-782

17. Seol, W., Choi, H. S., and Moore, D. D. (1995) Isolation of proteins that interact specifically with the retinoid $X$ receptor: two novel orphan receptors. Mol. Endocrinol. 9, 72-85

18. Reddy, A. P., Chen, J.-Y., Zacharewski, T., Gronemeyer, H., Voorhees, J. J., and Fisher, G. J. (1992) Characterization and purification of human retinoic acid receptor- $\gamma 1$ overexpressed in the baculovirus-insect cell system. Biochem. J. 287, 833-840

19. Allenby, G., Bocquel, M.-T., Saunder, M., Kazmer, S., Speck, J., Rosenberger, M., Lovey, A., Kastner, P., Grippo, J. F., Chambon, P., and Levin, A. A. (1993) Retinoic acid receptors and retinoid X receptors: Interaction with endogenous retinoic acids. Proc. Natl. Acad. Sci. USA 90, 30-34

20. Duell, E. A., Kang, S., and Voorhees, J. J. (1996) Retinoic acid isomers applied to human skin in vivo each induce a 4-hydroxylase that inactivates only trans retinoic acid. J. Invest. Dermatol. 106, 316-320

21. Fisher, G. J., Reddy, A. P., Datta, S. C., Kang, S., Yi, J. Y., Chambon, P., and Voorhees, J. J. (1995) All-trans retinoic acid induces cellular retinol-binding protein in vivo. J. Invest. Dermatol. 105, 80-86

22. Kurokawa, R., DiRenzo, J., Boehm, M., Sugarman, J., Gloss, B., Rosenfeld, M. G., Heyman, R. A., and Glass, C. K. (1994) Regulation of retinoid signalling by receptor polarity and allosteric control of ligand binding. Nature (London) 371, 528-531

23. Gendimenico, G. J., Stim, T. B., Corbo, M., Janssen, B., and Mezick, J. A. (1994) A pleiotropic response is induced in F9 embryonal carcinoma cells and rhino mouse skin by all-trans-retinoic acid, a RAR agonist but not by SR11237, a RXR-selective agonist. J. Invest. Dermatol. 102, 676-680

24. Armstrong, R. B., Ashenfelter, K. O., Eckhoff, C., Levin, A. A., and Shapiro, S. S. (1994) In The Retinoids: Biology, Chemistry, and Medicine (Sporn, M. B., Roberts, A. B., Goodman, D. S., eds) pp. 545-572, Raven Press, New York

25. Sanquer, S., and Gilchrest, B. A. (1994) Characterization of human cellular retinoic acid-binding proteins-I and -II: ligand binding affinities and distribution in skin. Arch. Biochem. Biophys. 311, 86-94

26. Astrom, A., Tavakkol, A., Pettersson, U., Cromie, M., Elder, J. T., and Voorhees, J. J. (1991) Molecular cloning of two human cellular retinoic acid-binding proteins (CRABP). Retinoic acid-induced expression of CRABP-II but not CRABP-I in adult human skin in vivo and in skin fibroblasts in vitro. J. Biol. Chem. 266, 17662-17666

27. Rosenthal, D. S., Griffiths, C. E. M., Yuspa, S. H., Roop, D. R., and Voorhees, J. J. (1992) Acute or chronic topical retinoic acid treatment of human skin in vivo alters the expression of epidermal transglutaminase, loricrin, involucrin, filaggrin, and keratins 6 and 13 but not keratins 1, 10, and 14. J. Invest. Dermatol. 98, 343-350

28. Rosenthal, D.S., Roop, D. R., Huff, C. A., Weiss, J. S., Ellis, C. N., Hamilton, T., and Voorhees, J. J. (1990) Changes in photo-aged human skin following topical application of all-trans retinoic acid.J. Invest. Dermatol. 95, 510-515

29. Astrom, A., Pettersson, U., Chambon, P., and Voorhees, J. J. (1994) Retinoic acid induction of human cellular retinoic acid-binding protein-II gene transcription is mediated by retinoic acid receptor-retinoid $\mathrm{X}$ receptor heterodimers bound to one far upstream retinoic acid-response element with 5-base pair spacing. J. Biol. Chem. 269, 22334-22339

30. Husmann, M., Hoffmann, B., Stump, G. D., Chytil, F., and Pfahl, M. (1992) A retinoic acid response element from the rat CRBPI promoter is activated by an RAR/RXR heterodimer. Biochem. Biophys. Res. Commun. 184, 1558-1564

31. Smith, W. C., Nakshatri, H., Leroy, P., Rees, J., and Chambon, P. (1991) A retinoic acid response element is present in the mouse cellular-retino binding protein 1 (CRBPI) promoter. EMBO J. 10, 2223-2230
32. Jiang, C. K., Magnaldo, T., Ohtsuki, M., Freedberg, I. M., and Blumberg, M. (1993) F.GF and TGF $\alpha$ specifically induce the activation- and hyperproliferation-associated keratins K\#6 and K\# 16. Proc. Nall. Acad. Sci. USA 90 , $6786-6790$

33. Navarro, J. M., Casatorres, J., and Jorcano, J. L. (1995) Elements controlling the expression and induction of the skin hyperproliferation-associated keratin K6. J. Biol. Chem. 270, 21362-21367

34. Chen, J. Y., Penco, S., Ostrowski, J., Balaguer, P., Pons, M., Starrett, J. E., Reczek, P., and Chambon, P. (1995) RAR-specific agonist/antagonists which dissociate transactivation and AP-1 transrepression inhibit anchorage-independent cell proliferation. Embo J. 14, 1187-1197

35. Saatcioglu, F., Claret, F. X., and Karin, M. (1994) Negative transcriptional regulation by nuclear receptors. Semin. Cancer Biol. 5, 347-359

36. Pfahl, M. (1993) Nuclear receptor/AP-1 interaction. Endocr. Rev. 14, $651-658$

37. Kligman, L. H., and Kligman, A. M. (1986) The nature of photoaging: its prevention and repair. Photodermatology 3, 215-227

38. Wlaschek, M., Heinen, G., Poswig, A., Schwarz, A., Krieg, T., and Scharfetter-Kochanek, K. (1994) UVA-induced autocrine stimulation of fibroblastderived collagenase/MMP-1 by interrelated loops of interleukin-1 and interleukin-6. Photochem. Photobiol. 59, 550-556

39. Fisher, G. J., Datta, S. C., Talwar, H. T., Wang, Z.-Q., Varani, J., Kang, S. and Voorhees, J. J. (1996) Molecular basis of sun-induced premature skin ageing and retinoid antagonism. Nature (London) 379, 335-339

40. Rafal, E. S., Griffiths, C. E. M., Ditre, C. M., Finkel, L. J., Hamilton, T. A., Ellis, C. N., and Voorhees, J. J. (1992) Topical tretinoin (retinoic acid) treatment for liver spots associated with photodamage. N. Engl. J. Med. 326, 368-374

41. Griffiths, C. E. M., Russman, A. N., Majmudar, G., Singer, R. S., Hamilion, T. A., and Voorhees, J. J. (1993) Restoration of collagen formation in photodamaged human skin by tretinoin (retinoic acid). N. Engl. J. Med. 329, 530-535

42. Schwartz, E., Cruickshank, F. A., Mezick, J. A., and Kligman, L. H. (1991) Topical all-trans retinoic acid stimulates collagen synthesis in vivo. J. Invest. Dermatol. 96, 975-978

43. Talwar, H. S., Griffiths, C. E. M., Fisher, G. J., Hamilton, T. A., and Voorhees, J. J. (1995) Reduced type I and type III procollagens in photodamaged adul human skin. J. Invest. Dermatol. 105, 285-290

44. Kang, S., Duell, E. A., Fisher, G. J., Datta, S. C., Wang, Z. Q., Reddy, A. P. Tavakkol, A., Yi, J. Y., Griffiths, C. E. M., Elder, J. T., and Voorhees, J. J. (1995) Application of retinol to human skin in vivo induces epidermal hyperplasia and cellular retinoid binding proteins characteristic of retinoic acid but without measurable retinoic acid levels or irritation. J. Invest. Dermatol. 105, 549-556

45. Kurlandsky, S. B., Duell, E. A., Kang, S., Voorhees, J. J., and Fisher, G. J. (1996) Auto-regulation of retinoic acid biosynthesis through regulation of retinol esterification in human keratinocytes. J. Biol. Chem. In Press

46. Torma, H., and Vahlquist, A. (1987) Retinol esterification by mouse epidermal microsomes: evidence for acyl-CoA:retinol acyltransferase activity. $J$. Invest. Dermatol. 88, 398-402

47. Siegenthaler, G., Saurat, J. H., and Ponec, M. (1990) Retinol and retinal metabolism. Biochem. J. 268, 371-378

47a. Vahlquist, A. (1982) Vitamin A in human skin: Detection and identification of retinoids in normal epidermis J. Invest. Dermatol. 79, 89-93

48. Kurlandsky, S. B., Xiao, J. H., Duell, E. A., Voorhees, J. J., and Fisher, G. J. (1994) Biological activity of all-trans retinol requires metabolic conversion to all-trans retinoic acid and is mediated through activation of nuclear retinoid receptors in human keratinocytes. J. Biol. Chem. 269, 32821-32827

49. Chen, S., Ostrowski, J., Whiting, G., Roalsvig, T., Hammer, L., Currier, S. J., Honeyman, J., Kwasniewski, B., Yu, K. L., and Sterzycki, R. (1995) Retinoic acid receptor gamma mediates topical retinoid efficacy and irritation in animal models. J. Invest. Dermatol. 104, 779-783

50. Duell, E. A., Derguini, F., Kang, S., Elder, J. T., and Voorhees, J. J. (1996) Extraction of human epidermis treated with retinol yields retro retinoids in addition to free retinol and retinyl esters. J. Invest. Dermatol. In Press

51. Buck, J., Derguini, F., Levi, E., Nakanishi, K., and Hammerling, U. (1991) Intracellular signaling by 14-hydroxy-4,14-retro-Science 254, 1654-1656

52. Duell, E. A., Astrom, A., Griffiths, C. E. M., Chambon, P., and Voorhees, J. J. (1992) Human skin levels of retinoic acid and cytochrome P-450-derived 4-hydroxyretinoic acid after topical application of retinoic acid in vivo to concentrations required to stimulate retinoic acid receptor-mediated transcription in vitro. J. Clin. Invest. 90, 1269-1274

53. Crettaz, M., Baron, A., Siegenthaler, G., and Hunziker, W. (1990) Ligand specificities of recombinant retinoic acid receptors $R A R \alpha$ and RAR Biochem. J. 272, 391-397

54. Reynolds, N. J., Fisher, G. J., Griffiths, C. E. M., Tavakkol, A., Talwar, H. S., Rowse, P. E., Hamilton, T. A., and Voorhees, J. J. (1993) Retinoic acid metabolites exhibit biological activity in human keratinocytes, mouse melanoma cells and hairless mouse skin in vivo. J. Pharmacol. Exp. Ther. 266, 1636-1642

55. Kang, S., Duell, E. A., Kim, K. J., and Voorhees, J. J. (1996) Liarozole inhibits human epidermal retinoic acid 4-hydroxylase activity and differentially augments human skin responses to retinoic acid and retinol in-vivo. J. Invest. Dermatol. In press 
56. Ong, D. E. (1994) Cellular transport and metabolism of Vitamin A: Roles of the cellular retinoid-binding proteins. Nutr. Rev. 52, S24-S31

57. Napoli, J. L. (1993) Biosynthesis and metabolism of retinoic acid: roles of CRBP and CRABP in retinoic acid homeostasis. J. Nutr. 123, 362-366

58. Fiorella, P. D., and Napoli, J. L. (1994) Microsomal retinoic acid metabolism. Effects of cellular retinoic acid-binding protein (type I) and C18-hydroxylation as an initial step. J. Biol. Chem. 269, 10538-10544

59. Posch, K. C., Bums, R. D., and Napoli, J. L. (1992) Biosynthesis of all-trans-retinoic acid from retinal. Recognition of retinal bound to cellular retinol binding protein (type I) as substrate by a purified cytosolic dehydrogenase.. J. Biol. Chem. 267, 19676-19682

60. Ottonello, S., Scita, G., Mantovani, G. Cavazzini, D., and Rossi, G. L. (1993) Retinol bound to cellular retinol-binding protein is a substrate for cytosolic retinoic acid synthesis. J. Biol. Chem. 268, 27133-27142

61. Donovan, M., Olofsson, B., Gustafson, A. L., Dencker, L., and Eriksson, U. (1995) The cellular retinoic acid binding proteins. J. Steroid Biochem. Mol. Biol. 53, 459-465

62. Chai, X., Boerman, M. H. E. M., Zhai, Y., and Napoli, J. L. (1995) Cloning of a cDNA for liver microsomal retinol dehydrogenase. J. Biol. Chem. 270, $3900-3904$

63. Chai, X., Zhai, Y., Popescu, G., and Napoli, J. L. (1995) Cloning of a cDNA for a second retinol dehydrogenase type II. Expression of its mRNA relative to type 1. J. Biol. Chem. 270, 28408-28412

64. Jetten, A. M. (1990) Multi-stage program of differentiation in human epidermal keratinocytes: regulation by retinoids. J. Invest. Dermatol. 95, 445-46S

65. Fuchs, E., and Green, H. (1981) Regulation of terminal differentiation of cultured human keratinocytes by vitamin A. Cell 25, 617-625

66. Asselineau, D., Dale, B. A., and Bernard, B. A. (1990) Filaggrin production by cultured human epidermal keratinocytes and its regulation by retinoic acid. Differentiation 45, 221-229

67. Yuspa, S. H., and Harris, C. C. (1974) Altered differentiation of mouse epidermal cells treated with retinyl acetate in vitro. Exp. Cell Res. 86, 95-105

68. Griffiths, C. E. M., Rosenthal, D. S., Reddy, A. P., Elder, J. T., Astrom, A., Leach, K., Wang, T. S., Finkel, L. J., Yuspa, S. H., and Voorhees, J. J. (1992) Short-term retinoic acid treatment increased in vivo, but decreases in vitro, epidermal transglutaminase- $\mathrm{K}$ enzyme activity and immunoreactivity. $J$. Invest. Dermatol. 99, 283-288

69. Magnaldo, T., Bernerd, F., Asselineau, D., and Darmon, M. (1992) Expression of loricrin is negatively controlled by retinoic acid in human epidermis reconstructed in vitro.Differentiation 49, 39-46

70. Hohl, D., Lichti, U., Breitkreutz, D., Steinert, P. M., and Roop, D. R. (1991) Transcription of the human loricrin gene in vitro is induced by calcium and cell density and suppressed by retinoic acid.. J. Invest. Dermatol. 96 , 414-418

71. Marvin, K. W., George, M. D., Fujimoto, F., Saunders, N. A., Bernacki, S. H., and Jetten, A. M. (1992) Cornifin, a cross-linked envelope precursor in keratinocytes that is down-regulated by retinoids. Proc. Natl. Acad. Sci. USA 89, 11026-11030

72. Futoryan, T., and Gilchrest, B. A. (1994) Retinoids and the skin. Nutr. Rev. $52,299-310$

73. Hardy, M. H. (1968) Clandular metaplasia of hair follicles and other responses to vitamin A excess in cultures of rodent skin. J. Embryol. Exp. Morphol. 19, 157-180
74. Aaronson, S. A., Bottare, D. P., Miki, T., Ron, D., Finch, P. W., Fleming, T. P., Ahn, J., Taylor, W. G., and Rubin, J. S. (1991) Keratinocyte growth factor: a fibroblast growth factor family member with unusual targert cell specificity. Ann. N.Y. Acad. Sci. 638, 62-77

75. Tavakkol, A., Elder, J. T., Griffiths, C. E. M., Cooper, K. D., Talwar, H. S., Fisher, G. J., Keane, K. M., Foltin, S. K., and Voorhees, J. J. (1992) Expression of growth hormone receptor, insulin-like growth factor (ICF-1) and IGF-1 receptor mRNA and proteins in human skin.. J. Invest. Dermatol. 99, 343-349

76. Schwartz, E., Mezick, J. A., Gendimenico, G. J., and Kligman, L. H. (1994) In vivo prevention of corticosteroid-induced skin atrophy by tretinoin in the hairless mouse is accompanied by modulation of collagen, glycosaminoglycans, and fibronectin. J. Invest. Dermatol. 102, 241-246

77. Dawson, M. I., Jong, L., Hobbs, P. D., Cameron, J. F., Chao, W. R., Pfahl, M., Lee, M. O., Shroot, B., and Pfahl, M. (1995) Conformational effects on retinoid receptor selectivity. Effects of retinoid bridging group on retinoid $X$ receptor activity and selectivity. J. Med. Chem. 38, 3368-3383

78. Lehmann, J. M., Jong, L., Fanjul, A., Cameron, J. F., Lu, X. P., Haefner, P., Dawson, M. I., and Prahl, M. (1992) Retinoids selective for retinoid X receptor response pathways. Science 258, $1944-1946$

79. Tavakkol, A., Griffiths, C. E. M., Keane, K. M., Palmer, R. D., and Voorhees, J. J. (1992) Cellular localization of mRNA for cellular retinoic acid-binding protein II and nuclear retinoic acid receptor-gamma $l$ in retinoic acid-treated human skin. J. Invest. Dermatol. 99, 146-150

80. Chen, S., Ostrowski, J., Whiting, G., Roalsvig, T., Hammer, L., Currier, S. J., Honeyman, J., Kwasniewski, B., Yu, K. L., and Sterzycki, R. (1995) Retinoic acid receptor gamma mediates topical retinoid efficacy and irritation in animal models. J. Invest. Dermatol. 104, 779-783

81. Scheinman R. I., Gualberto, A., Jewell, C. M., Cidlowshi, J. A., and Baldwin, A. S. J. (1995) Characterization of mechanisms involved in transrepression NF-kappa B by activated glucocorticoid receptors. Mol. Cell. Biol. 15, 943-953

82. Caldenhoven, E., Liden, J., Wissink, S., Van de Stolpe, A., Raaijmakers, J., Koenderman, L., Okret, S., Gustafsson, J., and Van der Saag, P. (1995) Negative cross-talk between RelA and the glucocorticoid receptor: a possible mechanism for the antiinflammatory action of glucocorticoids. Mol. Endocrinol. 9, 401-412

83. Fanjul, A., Dawson, M. I., Hobbs, P. D., Jong, L., Cameron, J. F., Harlev, E., Graupner, G., Lu, X. P., and Pfahl, M. (1994) A new class of retinoids with selective inhibition of AP-1 inhibits proliferation. Nature (London) 372, 107-111

84. Hirose, T., Apfel, R., Pfahl, M., and Jetten, A. M. (1995) The orphan receptor TAKI acts as a repressor of RAR-, RXR- and T3R-mediated signaling pathways. Biochem. Biophys. Res. Commun. 211 , 83-91

85. Cooney, A., Tsai, S., O'Malley, B., and Tsai, M. (1992) Chicken ovalbumin upstream promoter transcription factor (COUP-TF) dimers bind to different GGTCA response elements, allowing COUP-TF to repress hormonal induction of the vitamin $D_{3}$, thyroid hormone, and retinoic acid receptors. Mol. Cell. Biol. 12, 4153-4163

86. Gilfix, B. M., and Eckert, R. L. (1985) Coordinate control by vitamin A of keratin gene expression in human keratinocytes. J. Biol. Chem. 260, 14026-14029

87. Green, H., and Watt, F. M. (1982) Regulation by vitamin A of envelope cross-linking in cultured keratinocytes derived from different human epithelia. Mol. Cell Biol. 2, 1115-1117 